\title{
Two-way CSI-assisted AF relaying with HPA nonlinearity
}

Book or Report Section

Accepted Version

Qi, J., Aissa, S. and Alouini, M.-S. (2015) Two-way CSIassisted AF relaying with HPA nonlinearity. In: 2015 IEEE International Conference on Communications (ICC). IEEE, pp. 1643-1648. ISBN 9781467364324 doi:

https://doi.org/10.1109/ICC.2015.7248560 Available at http://centaur.reading.ac.uk/42900/

It is advisable to refer to the publisher's version if you intend to cite from the work. See Guidance on citing.

To link to this article DOI: http://dx.doi.org/10.1109/ICC.2015.7248560

Publisher: IEEE

All outputs in CentAUR are protected by Intellectual Property Rights law, including copyright law. Copyright and IPR is retained by the creators or other copyright holders. Terms and conditions for use of this material are defined in the End User Agreement. 


\section{www.reading.ac.uk/centaur}

\section{CentAUR}

Central Archive at the University of Reading

Reading's research outputs online 


\title{
Two-Way CSI-Assisted AF Relaying with HPA Nonlinearity
}

\author{
Jian $\mathrm{Qi}^{*}$, Sonia Aïssa ${ }^{\dagger}$, and Mohamed-Slim Alouini ${ }^{\ddagger}$ \\ ${ }^{*}$ University of Reading, Reading, Berkshire, UK. \\ $\dagger$ Institut National de la Recherche Scientifique (INRS), University of Quebec, Montreal, QC, Canada. \\ $\ddagger$ King Abdullah University of Science and Technology (KAUST), Thuwal, Makkah Province, Saudi Arabia. \\ Email: a.j.qi@ reading.ac.uk; aissa@emt.inrs.ca; slim.alouini@kaust.edu.sa
}

\begin{abstract}
In this paper, we investigate half-duplex two-way dual-hop channel state information (CSI)-assisted amplify-andforward (AF) relaying in the presence of high-power amplifier (HPA) nonlinearity at relays. The expression for the end-toend signal-to-noise ratio (SNR) is derived as per the modified system model by taking into account the interference caused by relaying scheme and HPA nonlinearity. The system performance of the considered relaying network is evaluated in terms of average symbol error probability (SEP) in Nakagami- $m$ fading channels, by making use of the moment-generating function (MGF) approach. Numerical results are provided and show the effects of several parameters, such as quadrature amplitude modulation (QAM) order, number of relays, HPA parameters, and Nakagami parameter, on performance.
\end{abstract}

\section{INTRODUCTION}

Cooperative relaying stands as a promising mechanism for future wireless communication networks, which can enhance transmission reliability, enlarge system capacity, broaden network coverage, and ensure quality of service (QoS). The nodes in the network could not only act as source node to issue information, but also perform as relay to forward the signals received from other nodes. In this paper, we investigate the half-duplex two-way amplify-and-forward (AF) relaying, where source nodes simultaneously transmit their information to the relay nodes in the first phase, and then, in the following phase, the relay nodes simply amplify the received signals and forward them to the source nodes. The two-way AF relaying can cope with the drawback of the one-way relaying and obtain higher spectrum efficiency [1], [2].

However, the practical performance of the dedicated twoway relaying is crucially affected by radio frequency (RF) impairments, which bring distortions to the network and then degrade the system performance [3], [4]. Herein, we consider one type of RF impairments, namely, high-power amplifier (HPA) nonlinearity. The HPA may operate in its nonlinear region., which causes in-band and out-of-band distortions [5], [6]. The nonlinear HPAs can be characterized by two kinds of models: memoryless models with frequency-flat responses, and memory models with frequency-selective responses. The former include Saleh model for travelling wave tube amplifier

M.-S. Alouini is a member of the KAUST SRI Center for Uncertainty Quantification in Computational Science and Engineering.
(TWTA), solid state power amplifier (SSPA) model, softenvelope limiter (SEL) model, and memoryless polynomial model. Memory models contain Volterra, Wiener, Hammerstein, Wiener-Hammerstein, and memory polynomial models. Recent research effort has studied the effect of HPA nonlinearity on the performance of orthogonal frequency-division multiplexing (OFDM)-based cooperative systems using the Bussgang's theorem in [7]. On the other hand, one-way dualhop AF relaying with HPA nonlinearity in semi-blind relays was investigated by making use of memoryless polynomial model for HPAs in the authors' previous work [8].

In this paper, we study the half-duplex two-way dual-hop channel state information (CSI)-assisted AF relaying system in the presence of HPA nonlinearity at relays. The updated model has been built up by taking into account the factors caused by nonlinear HPAs. The expression for the end-toend signal-to-noise ratio (SNR) has been modelled, and followed by two asymptotic expressions for low and high SNR regions, respectively. Then, by making use of the momentgenerating function (MGF) approach, the expression for the average symbol error probability (SEP) of the considered system is derived in Nakagami- $m$ fading channels. Afterwards, numerical results and comparison are provided and show the effects of several parameters, such as number of relays, HPA parameters, quadrature amplitude modulation (QAM) order, and Nakagami parameter, on the system performance.

The remainder of this paper is organized as follows: Section II introduces the half-duplex two-way dual-hop CSI-assisted AF relaying system model in the presence of HPA nonlinearity at the relays. The system performance metrics, in terms of end-to-end SNR and average SEP, are derived in Section III. Numerical results and comparisons are then presented in Section IV, followed by the conclusion in Section V.

\section{SySTEM MODEL}

We consider a dual-hop half-duplex two-way AF cooperative relaying system allocated with two source nodes, $S_{1}$ and $S_{2}$, and $N$ relay nodes, $R_{n}(n=1,2, \ldots, N)$, which are all equipped with a single antenna. No direct path between $S_{1}$ and $S_{2}$ exists so that the source nodes communicate through the relays. The transmission works in two phases, i.e., multipleaccess (MAC) phase and broadcast (BC) phase. In the first 
phase, $S_{1}$ and $S_{2}$ simultaneously transmit their information to the $N$ relay nodes. Then, in the second phase, the relays amplify the received signals and broadcast them to the source nodes.

In practice, the performance of the two-way relaying system may get affected by HPA nonlinearity, which causes in-band and out-of-band distortions. Herein, we only consider in-band distortion caused by nonlinear HPAs implemented at the relay nodes. In particular, the memoryless polynomial model [9], [10] is used here to describe the characteristics of nonlinear HPA. In a general way, the input-output relationship of the nonlinear HPA can be expressed as

$$
y_{\text {out }}(t)=x_{\text {in }}(t) \sum_{k=0}^{K} a(2 k+1)\left|x_{\text {in }}(t)\right|^{2 k},
$$

where $x_{\text {in }}(t)$ and $y_{\text {out }}(t)$ denote the baseband-equivalent input and output signals, respectively, $K$ is the order of the polynomial function, and $\{a(2 k+1)\}$ refers to a set of coefficients. Note only odd-order product terms in (1) are considered due to the fact that the signals generated from the even-order terms are outside the frequency band of interest [10]. For simplicity of the analysis, the HPA coefficients are assumed to be perfectly known.

In the MAC phase of the two-way relaying system, the received baseband-equivalent signal at the $n$-th $(n=$ $1,2, \ldots, N)$ relay node is given by

$y_{r_{n}}(t)=\sqrt{P_{s_{1}}} x_{1}(t) \otimes h_{n}(t)+\sqrt{P_{s_{2}}} x_{2}(t) \otimes g_{n}(t)+n_{r_{n}}(t)$,

where $x_{1}(t)$ and $x_{2}(t)$ denote the normalized basebandequivalent transmitted signal from the source nodes $S_{1}$ and $S_{2}$, respectively, $P_{s_{1}}$ and $P_{s_{2}}$ denote the transmit power at $S_{1}$ and $S_{2}$, respectively, $h_{n}(t)$ and $g_{n}(t)$ are the channel coefficients from the sources $S_{1}$ and $S_{2}$ to the $n$-th relay node, respectively, $n_{r_{n}}(t)$ refers to the noise term at the $n$-th relay, belonging to $\mathcal{C N}\left(0, N_{0}\right)$ and uncorrelated with the transmitted signals, and $\otimes$ denotes the convolution product.

In the BC phase, the received signal is multiplied by the gain of the relay, $G_{n}(t)$, which can be expressed as

$$
G_{n}(t)=\sqrt{\frac{P_{n}}{P_{s_{1}}\left|h_{n}(t)\right|^{2}+P_{s_{2}}\left|g_{n}(t)\right|^{2}+N_{0}}},
$$

where $P_{n}$ represents the power of the $n$-th relay. Then, the signal at the output of HPA is given by

$$
x_{r_{n}}(t)=G_{n}(t) y_{r_{n}}(t) \sum_{k=0}^{K_{n}} a_{n}(2 k+1)\left|G_{n}(t) y_{r_{n}}(t)\right|^{2 k},
$$

where $K_{n}$ and $\left\{a_{n}(2 k+1)\right\}$ represent the order and coefficient set of the polynomial model for the HPA at the $n$-th relay node, respectively.

It is assumed that the channels between the sources and relays are reciprocal, and remain constant during the MAC and $\mathrm{BC}$ phases. Without loss of the generality, we consider the received signal at the source $S_{2}$. In the BC phase, using the CSI-assisted AF relaying, the received signal at the source node $S_{2}$ from the $i$-th relay is given by

$$
y_{i, s_{2}}(t)=x_{r_{n}}(t) \otimes g_{n}(t)+n_{i, s_{2}}(t),
$$

where $n_{i, s_{2}}(t)$ denotes the noise at the source node $S_{2}$ from the $n$-th relay, belonging to $\mathcal{C N}\left(0, N_{0}\right)$.

Remark 1: Unlike the case with linear HPAs, the selfinterference presenting the source nodes cannot be removed totally due to the nonlinear characteristics of the HPAs at the relays, even though the perfect CSI is assumed to be known at the sources, which consequently degrades the performance of two-way relaying.

It is assumed that perfect sampling is performed in order to profit from the property of the raise-cosine rolloff and rootraised cosine rolloff pulses. Based on this, substituting (4) into (5) and omitting the time index, the received signal at the source node $S_{2}$ from the $i$-th relay can be expressed as

$$
\begin{aligned}
& y_{i, s_{2}}=n_{i, s_{2}}+g_{n} G_{n}\left(\sqrt{P_{s_{1}}} x_{1} h_{n}+\sqrt{P_{s_{2}}} x_{2} g_{n}+n_{r_{n}}\right) \\
& \times \sum_{k=0}^{K_{n}} a_{n}(2 k+1)\left|G_{n}\left(\sqrt{P_{s_{1}}} x_{1} h_{n}+\sqrt{P_{s_{2}}} x_{2} g_{n}+n_{r_{n}}\right)\right|^{2 k} .
\end{aligned}
$$

By further subtracting the self-interference properly, we can obtain the expression as

$$
\begin{aligned}
& y_{i, s_{2}}^{F}=a_{n}(1) \sqrt{P_{s_{1}}} G_{n} h_{n} g_{n} x_{1}+n_{i, s_{2}}+a_{n}(1) G_{n} g_{n} n_{r_{n}} \\
& +g_{n} G_{n}\left(\sqrt{P_{s_{1}}} h_{n} x_{1}+\sqrt{P_{s_{2}}} g_{n} x_{2}+n_{r_{n}}\right) \\
& \times \sum_{k=1}^{K_{n}} a_{n}(2 k+1)\left|G_{n}\left(\sqrt{P_{s_{1}}} h_{n} x_{1}+\sqrt{P_{s_{2}}} g_{n} x_{2}+n_{r_{n}}\right)\right|^{2},
\end{aligned}
$$

where the interference term comes from both the HPA nonlinearity and AF relaying. The self-interference could be removed for $k=0$, while it cannot be mitigated for the terms with $k \geq 1$. The effective end-to-end SNR via the $n$-th relay, $\gamma_{n}^{g}$, is given by (8), where $E[\cdot]$ denotes the expectation operator. It is not straightforward to derive the SNR expression for the case with large values of the HPA parameter $K_{n}$ in closedform. Herein, for simplicity of the analysis, we study the case with $K_{n}=1$, i.e., 3 -order polynomial model for the nonlinear HPAs. Thus, the effective end-to-end SNR becomes (9), where $\operatorname{Re}[\cdot]$ refers to the real part of a complex number.

\section{IMPACT OF HPA NONLINEARITY}

In this section, we investigate the impact of HPA nonlinearity on the performance of the considered two-way CSI-assisted AF relaying system in Nakagami- $m$ fading environment.

In a generic way, the probability density function (PDF) of the received SNR in Nakagami- $m$ fading channels can be expressed as

$$
f_{\gamma}(\gamma)=\frac{m^{m}}{\Gamma(m) \bar{\gamma}^{m}} \gamma^{m-1} \exp \left(-\frac{m}{\bar{\gamma}} \gamma\right),
$$

where $\bar{\gamma}$ refers to the average received SNR, $\Gamma(\cdot)$ denotes 


$$
\begin{aligned}
\gamma_{n}^{g}=E & {\left[\left|a_{n}(1) \sqrt{P_{s_{1}}} G_{n} h_{n} g_{n} x_{1}\right|^{2}\right] /\left\{N_{0}+E\left[\mid a_{n}(1) G_{n} g_{n} n_{r_{n}}\right.\right.} \\
& \left.\left.+\left.g_{n} G_{n}\left(\sqrt{P_{s_{1}}} h_{n} x_{1}+\sqrt{P_{s_{2}}} g_{n} x_{2}+n_{r_{n}}\right) \sum_{k=1}^{K_{n}} a_{n}(2 k+1)\left|G_{n}\left(\sqrt{P_{s_{1}}} h_{n} x_{1}+\sqrt{P_{s_{2}}} g_{n} x_{2}+n_{r_{n}}\right)\right|^{2 k}\right|^{2}\right]\right\} .
\end{aligned}
$$

$$
\begin{aligned}
\gamma_{n}= & P_{s_{1}}\left|a_{n}(1) G_{n} h_{n} g_{n}\right|^{2} /\left\{N_{0}+E\left[\mid a_{n}(1) G_{n} g_{n} n_{r_{n}}\right.\right. \\
& \left.\left.+\left.a_{n}(3) g_{n} G_{n}^{3}\left(\sqrt{P_{s_{1}}} h_{n} x_{1}+\sqrt{P_{s_{2}}} g_{n} x_{2}+n_{r_{n}}\right)\left|\left(\sqrt{P_{s_{1}}} h_{n} x_{1}+\sqrt{P_{s_{2}}} g_{n} x_{2}+n_{r_{n}}\right)\right|^{2}\right|^{2}\right]\right\} .
\end{aligned}
$$

$$
\begin{aligned}
\gamma_{n, 1}= & \frac{P_{s_{1}} P_{n} N_{0}^{2}\left|a_{n}(1) h_{n} g_{n}\right|^{2}}{\left(P_{s_{1}}\left|h_{n}\right|^{2}+P_{s_{2}}\left|g_{n}\right|^{2}+N_{0}\right) N_{0}^{3}+P_{n} E\left[\left.\left.\left|a_{n}(1) N_{0} g_{n} n_{r_{n}}+a_{n}(3) g_{n} P_{n} n_{r_{n}}\right| n_{r_{n}}\right|^{2}\right|^{2}\right]} \\
= & P_{s_{1}} P_{n} N_{0}^{2}\left|a_{n}(1) h_{n} g_{n}\right|^{2} /\left\{\left(P_{s_{1}}\left|h_{n}\right|^{2}+P_{s_{2}}\left|g_{n}\right|^{2}+N_{0}\right) N_{0}^{3}\right. \\
& \left.+P_{n}\left[N_{0}\left|a_{n}(1) g_{n}\right|^{2}+\left|a_{n}(3) g_{n} P_{n}\right|^{2} E\left(\left|n_{r_{n}}\right|^{6}\right)+2 \operatorname{Re}\left(a_{n}(1)\left(a_{n}(3)\right)^{*} P_{n} N_{0}\left|g_{n}\right|^{2}\right) E\left(\left|n_{r_{n}}\right|^{4}\right)\right]\right\} .
\end{aligned}
$$

the Gamma function, and $m \geq 1 / 2$ is the Nakagami parameter representing the average fading power. Denote $\gamma_{s_{1}, r_{n}}=\frac{P_{s_{1}}\left|h_{n}\right|^{2}}{N_{0}}, \gamma_{s_{2}, r_{n}}=\frac{P_{s_{2}}\left|g_{n}\right|^{2}}{N_{0}}$, and $\gamma_{r_{n}, s_{2}}=\frac{P_{r_{i}}\left|g_{n}\right|^{2}}{N_{0}}$ as the received SNRs from the source nodes $S_{1}$ and $S_{2}$ to the $n$-th relay, and from the $n$-th relay to the source $S_{2}$, whose PDFs are of the form (10), with Nakagami parameters $m_{s_{1}, r_{n}}, m_{s_{2}, r_{n}}, m_{r_{n}, s_{2}}$ and expectations $\bar{\gamma}_{s_{1}, r_{n}}=m_{s_{1}, r_{n}} \frac{P_{s_{1}}}{N_{0}}$, $\bar{\gamma}_{s_{2}, r_{n}}=m_{s_{2}, r_{n}} \frac{P_{s_{2}}}{N_{0}}, \bar{\gamma}_{r_{n}, s_{2}}=m_{r_{n}, s_{2}} \frac{P_{r_{i}}}{N_{0}}$, respectively. Note that $m_{s_{2}, r_{n}}=m_{r_{n}, s_{2}}$ by assuming the channel reciprocity.

As noticed from (9), the end-to-end SNR expression for the case with $K_{n}=1$ remains complicated for the purpose of expression derivation in closed-form. In order to achieve appropriate analytical expressions, we investigate two asymptotic scenarios.

Scenario 1: The noise dominates the interface term, i.e., $\left|n_{r_{n}}\right| \gg\left|\sqrt{P_{s_{1}}} h_{n} x_{1}+\sqrt{P_{s_{2}}} g_{n} x_{2}\right|$. In this case, the system works in the low SNR region. Accordingly, the end-to-end SNR can be expressed as (11), where the even-order moments of the Gaussian-distributed random variable $\left|n_{\mathrm{s}, \mathrm{r}_{n}}\right|$ are given by

$$
E\left[\left|n_{\mathrm{r}_{n}}\right|^{p}\right]=N_{0}^{\frac{p}{2}}(p-1) ! ! \quad \text { if } p \text { is even, }
$$

where $(q)$ !! denotes the double factorial, that is the product of every odd number from $q$ to 1 , if $q$ is odd. Then, we have

$$
\begin{aligned}
& E\left[\left|n_{\mathrm{r}_{n}}\right|^{2}\right]=N_{0}, \\
& E\left[\left|n_{\mathrm{r}_{n}}\right|^{4}\right]=3 N_{0}^{2}, \\
& E\left[\left|n_{\mathrm{r}_{n}}\right|^{6}\right]=15 N_{0}^{3} .
\end{aligned}
$$

Substituting (13) into (11), the end-to-end SNR via the $n$-th relay in the low SNR region can be further expressed as (14).
Scenario 2: We consider the case that the system works in the high SNR region. The noise could be neglected compared to the interface term caused by the transmitted signal, i.e., $\left|n_{r_{n}}\right| \ll\left|\sqrt{P_{s_{1}}} h_{n} x_{1}+\sqrt{P_{s_{2}}} g_{n} x_{2}\right|$. In this case, the end-toend SNR can be expressed as

$$
\begin{aligned}
& \gamma_{n, 2}=P_{s_{1}}\left|a_{n}(1) h_{n}\right|^{2} /\left\{E \left[\mid a_{n}(1) n_{r_{n}}+a_{n}(3) G_{n}^{2}\right.\right. \\
& \cdot\left(\sqrt{P_{s_{1}}} h_{n} x_{1}+\sqrt{P_{s_{2}}} g_{n} x_{2}\right) \\
& \left.\left.\left.\cdot\left|\left(\sqrt{P_{s_{1}}} h_{n} x_{1}+\sqrt{P_{s_{2}}} g_{n} x_{2}\right)\right|^{2}\right|^{2}\right]\right\}
\end{aligned}
$$

By further performing the approximation, the expression could be further derived as (16), which could be further approximated as

$$
\begin{aligned}
\gamma_{n, 2} \approx & \left|a_{n}(1)\right|^{2} P_{s_{1}}\left|h_{n}\right|^{2}\left(P_{s_{1}}\left|h_{n}\right|^{2}+P_{s_{2}}||^{2}\right)^{2} \\
& \div\left\{\left|a_{n}(1)\right|^{2} N_{0}\left(P_{s_{1}}\left|h_{n}\right|^{2}+P_{s_{2}}\left|g_{n}\right|^{2}\right)^{2}\right. \\
& \left.+\left|a_{n}(3)\right|^{2} P_{n}^{2}\left(\left|\sqrt{P_{s_{1}}} h_{n}\right|+\left|\sqrt{P_{s_{2}}} g_{n}\right|\right)^{6}\right\} .
\end{aligned}
$$

At the destination node, the maximal ratio combining (MRC) is used to utilise the signals received at the from the source and the $N$ relays. As per the combination scheme, the end-to-end SNR at the output of the MRC detector in the presence of HPA nonlinearity is given by

$$
\gamma_{\text {tot }}=\sum_{i=1}^{N} \gamma_{n} .
$$

Applying the MGF approach, the average SEP of $M$-QAM 


$$
\gamma_{n, 1}=\frac{\left|a_{n}(1)\right|^{2} \gamma_{s_{1}, r_{n}} \gamma_{r_{n}, s_{2}}}{1+\gamma_{s_{1}, r_{n}}+\gamma_{s_{2}, r_{n}}+\gamma_{r_{n}, s_{2}}\left\{\left|a_{n}(1)\right|^{2}+15\left|a_{n}(3) P_{n}\right|^{2}+6 \operatorname{Re}\left[a_{n}(1)\left(a_{n}(3)\right)^{*} P_{n}\right]\right\}}
$$

$$
\begin{aligned}
\gamma_{n, 2} & \approx \frac{P_{s_{1}}\left|a_{n}(1) h_{n}\right|^{2}}{\left|a_{n}(1)\right|^{2} N_{0}+\left|a_{n}(3) G_{n}^{2}\right|^{2} E\left\{\left|\left(\sqrt{P_{s_{1}}} h_{n} x_{1}+\sqrt{P_{s_{2}}} g_{n} x_{2}\right)\right|^{6}\right\}} \\
& =\frac{\left|a_{n}(1)\right|^{2} P_{s_{1}}\left|h_{n}\right|^{2}\left(P_{s_{1}}\left|h_{n}\right|^{2}+P_{s_{2}}\left|g_{n}\right|^{2}\right)^{2}}{\left|a_{n}(1) g_{n}\right|^{2} N_{0}\left(P_{s_{1}}\left|h_{n}\right|^{2}+P_{s_{2}}\left|g_{n}\right|^{2}\right)^{2}++\left|a_{n}(3)\right|^{2} P_{n}^{2} E\left[\left|\left(\sqrt{P_{s_{1}}} h_{n} x_{1}+\sqrt{P_{s_{2}}} g_{n} x_{2}\right)\right|^{6}\right]} .
\end{aligned}
$$

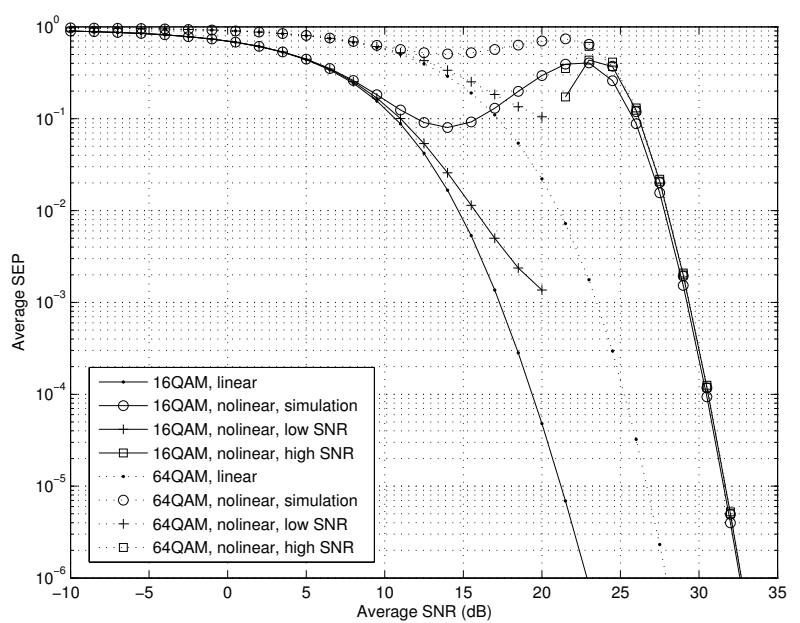

Fig. 1: Average SEP versus average SNR for different modulation formats.

in the two-way CSI-assisted AF cooperative system with HPA nonlinearity at the relays can be expressed as

$$
\begin{aligned}
P_{s} & =\frac{4}{\pi} a_{\mathrm{QAM}} \int_{0}^{\pi / 2} \prod_{i=1}^{N} M_{\gamma_{n}}\left(-\frac{g_{\mathrm{QAM}}}{\sin ^{2} \theta}\right) d \theta \\
& -\frac{4}{\pi} a_{\mathrm{QAM}}^{2} \int_{0}^{\pi / 4} \prod_{i=1}^{N} M_{\gamma_{n}}\left(-\frac{g_{\mathrm{QAM}}}{\sin ^{2} \theta}\right) d \theta
\end{aligned}
$$

where $a_{\mathrm{QAM}}=1-1 / \sqrt{M}, g_{\mathrm{QAM}}=3 /[2(M-1)]$, and $M_{Z}(s)=E[\exp (s Z)]$ denotes the MGF of random variable. Note that deriving the expression, $M_{\gamma_{n}}(s)$, in closed-form is not straightforward. The expression for the average SEP of $M$-QAM in the two-way CSI-assisted AF cooperative relaying system in the presence of HPA nonlinearity, over Nakagami$m$ fading channels, is obtained using numerical integration method.

\section{Numerical Results}

In this section, we demonstrate numerical results to show the effect of nonlinear HPAs at the relays on the performance of

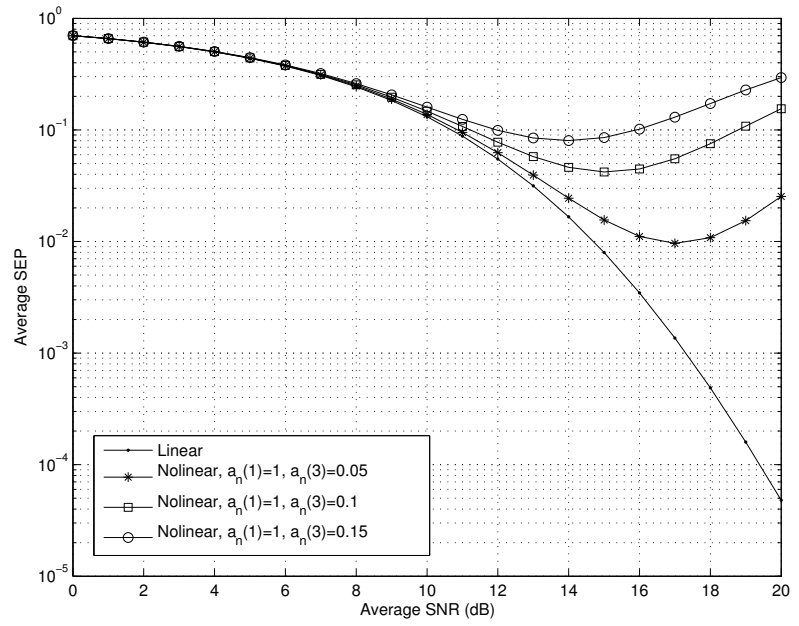

Fig. 2: Average SEP versus average SNR for different values of HPA coefficients $\left\{a_{n}(2 k+1)\right\}_{k=0}^{1}$.

half-duplex two-way dual-hop CSI-assisted AF relaying system in Nakagami- $m$ fading environment, in terms of average SEP. It is assumed that the power at the source nodes are equal to those at the relays, i.e., $P_{s_{1}}=P_{s_{2}}=P_{n}(n=1,2, \ldots, N)$. We define the average SNR as $\bar{\gamma}=\frac{P_{n}}{N_{0}}$. Unless otherwise specified, we set the Nakagami parameters at all links to be 2, i.e., $m_{s_{1}, r_{n}}=m_{s_{2}, r_{n}}=m_{r_{n}, s_{2}}=2(n=1,2, \ldots, N)$, and the HPA parameters at all relays to be $\left\{a_{n}(2 k+1)\right\}_{k=0}^{1}=$ $\{1,0.15\}(n=1,2, \ldots, N)$.

First, we evaluate the average SEP versus average SNR for 16QAM and 64QAM with $N=3$ relays. Fig. 1 shows that there is a huge degradation of the average SEP in the case with HPA nonlinearity, especially in the average SNR region $10 \mathrm{~dB}-25 \mathrm{~dB}$, which occurs due to the nonlinear distortion. In this specific range, the average SEP cannot be reduced by increasing the average SNR, where the deconstructive effect of larger SNR, i.e., higher distortion, outperforms the constructive impact. On the other hand, in the SNR region higher than $25 \mathrm{~dB}$, the average SEP will decrease along with the enlarged average SNR. Furthermore, as observed from the figure, the asymptotic performance at low and high SNR regions, plotted 


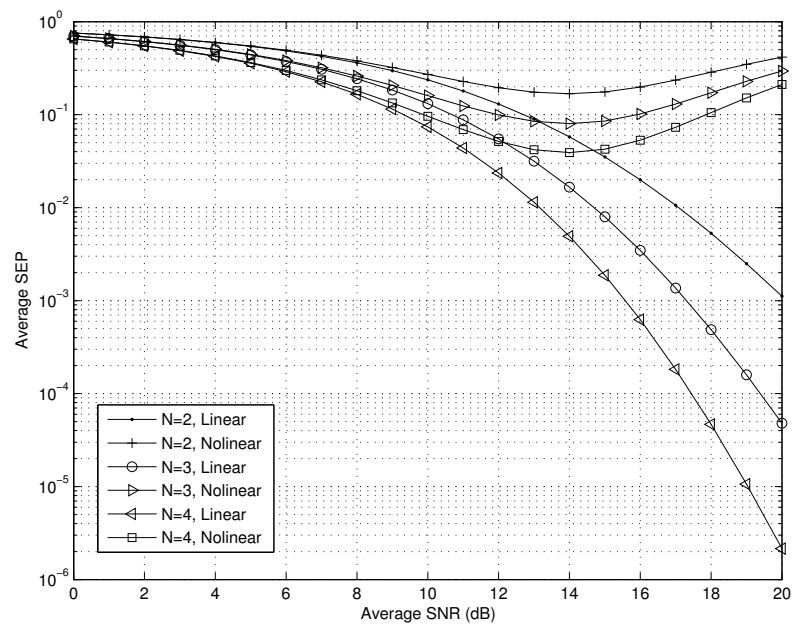

Fig. 3: Average SEP versus average SNR for different numbers of relays.

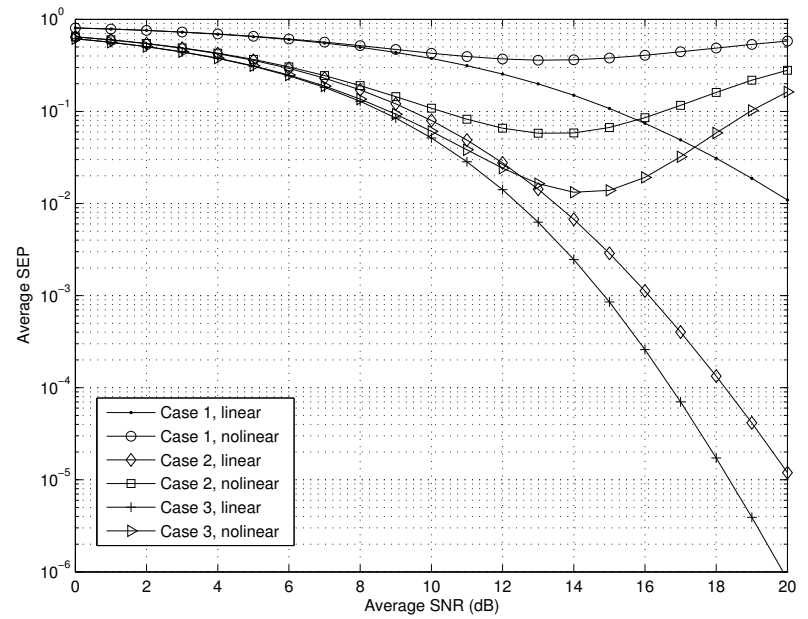

Fig. 4: Average SEP versus average SNR for different values of Nakagami parameters.

according to the derived expressions, matches very well with the simulation results, especially for the case with average SNR lower than $10 \mathrm{~dB}$ and high than $23 \mathrm{~dB}$.

In Fig. 2, we examine the average SEP versus the average SNR for different values of HPA coefficients for the case with $N=3$ relays, considering 16-QAM as the modulation scheme. Three scenarios of the HPA coefficients are investigated herein: (1) $\left\{a_{n}(2 k+1)\right\}_{k=0}^{1}=\{1,0.05\}(n=1,2, \ldots, N)$; (2) $\left\{a_{n}(2 k+1)\right\}_{k=0}^{1}=\{1,0.1\}(n=1,2, \ldots, N)$; (3) $\left\{a_{n}(2 k+1)\right\}_{k=0}^{1}=\{1,0.15\}(n=1,2, \ldots, N)$. The results demonstrate that the average SEP decreases in the case with smaller values of $a_{n}(3)$, profiting from the less distortion caused by nonlinear HPAs.

Fig. 3 demonstrates the impact of the relay number, $N$, on the average SEP of the considered two-way relaying network by using 16-QAM as the modulation scheme. It can be noticed that there is a slight decrease on the average SEP by increasing the number of relays in the case with HPA nonlinearity. However, it does not help a lot, especially in the high SNR region due to the severe distortion caused by HPA nonlinearity. The diversity order cannot be effectively achieved by increasing the number of relays unless appropriate compensation scheme for HPA nonlinearity is applied in the system.

Finally, in Fig. 4, we illustrate the average SEP versus average SNR for different cases of Nakagami parameters in the presence of HPA nonlinearity, with 16-QAM and $N=3$. We consider three cases for the Nakagami parameters: (1) $m_{s_{1}, r_{n}}=m_{s_{2}, r_{n}}=m_{r_{n}, s_{2}}=1(n=1,2, \ldots, N)$; (2) $m_{s_{1}, r_{n}}=2, m_{s_{2}, r_{n}}=m_{r_{n}, s_{2}}=4(n=1,2, \ldots, N)$; (3) $m_{s_{1}, r_{n}}=4, m_{s_{2}, r_{n}}=m_{r_{n}, s_{2}}=2(n=1,2, \ldots, N)$. It can be observed that the average SEP of the two-way relaying system becomes lower in the case with higher values of Nakagami parameters. Moreover, the link from the other source node to the relays plays a more crucial role on the performance at the targeted source node, that is, high values of $m_{s_{1}, r_{n}}$ bring a better system performance, which is validated by comparing cases (2) and (3).

\section{CONClusion}

In this paper, half-duplex two-way dual-hop CSI-assisted $\mathrm{AF}$ relaying systems in the presence of nonlinear HPAs at the relays were studied. We calculated the expression for the end-to-end SNR and derived closed-form expression for two asymptotic cases at low and high SNR regions, respectively. The performance of the considered two-way relaying system was evaluated in terms of average SEP in Nakagami- $m$ fading environment, by making use of the MGF scheme. Numerical results and comparison were also provided and show the effects of the HPA parameters, numbers of relays, QAM order, Nakagami parameters, on the system performance. Ongoing research is to propose an iterative compensation mechanism for HPA nonlinearity in two-way relaying systems.

\section{REFERENCES}

[1] B. Rankov and A. Wittneben, "Spectral efficient protocols for halfduplex fading relaying channels," IEEE J. Select. Areas Commun., vol. 25, no. 2, pp. 379-389, Feb. 2007.

[2] Y. Han, S. H. Ting, C. K. Ho, and W. H. Chin, "Performance bounds for two-way amplify-and-forward relaying," IEEE Trans. Wireless Commun., vol. 8, no. 1, pp. 432-439, Jan. 2009.

[3] J. Qi, S. Aïssa, and M.-S. Alouini, "Dual-hop amplify-and-forward cooperative relaying in the presence of Tx and Rx in-phase and quadraturephase imbalance," IET Commun., vol. 8, no. 3, pp. 287-298, Feb. 2014.

[4] J. Qi and S. Aïssa, "On the power amplifier nonlinearity in MIMO transmit beamforming systems," IEEE Trans. Commun., vol. 60, no. 3 , pp. 876-887, Mar. 2012.

[5] D. Dardari and V. T. A. Vaccari, "A theoretical characterization of nonlinear distortion effects in OFDM systems," IEEE Trans. Commun., vol. 48 , no. 10 , pp. $1755-1764$, Oct. 2000.

[6] J. Qi and S. Aïssa, "Analysis and compensation of power amplifier nonlinearity in MIMO transmit diversity systems," IEEE Trans. Veh. Technol., vol. 59, no. 6, pp. 2921-2931, July 2010.

[7] V. del Razo, T. Riihonen, F. Gregorio, S. Werner, and R. Wichman, "Nonlinear amplifier distortion in cooperative amplify-and-forward OFDM systems," in Proc. IEEE Wireless Commun. and Networking Conf. (WCNC'09), Budapest, Hungary, Apr. 2009. 
[8] J. Qi, S. Aïssa, and M.-S. Alouini, "Performance analysis of AF cooperative systems with HPA nonlinearity in semi-blind relays," in Proc. IEEE Global Telecommun. Conf. (Globecom'12), Anaheim, USA, Dec. 2012, pp. 4387-4391.

[9] S. Benedetto, E. Biglieri, and R. Daffara, "Modeling and performance evaluation of nonlinear satellite links-a Volterra series approach," IEEE Trans. Aerosp. Electron. Syst., vol. 15, no. 4, pp. 494-507, July 1979.

[10] G. T. Zhou, H. Qian, L. Ding, and R. Raich, "On the baseband representation of a bandpass nonlinearity," IEEE Trans. Signal Process., vol. 53, no. 8, pp. 2953-2957, Aug. 2005. 